\title{
Exploring Extinction and Structure in the Milky Way Disk With 2MASS and Spitzer
}

\author{
G. Zasowski, S. R. Majewski, D. L. Nidever and R. Indebetouw \\ Department of Astronomy, University of Virginia \\ Box 400325, Charlottesville, VA, 22904 USA \\ email: gailis@virginia.edu
}

\begin{abstract}
We present new maps of the distribution of both dust and stars across the Galactic disk, based largely on an improved analysis of 2MASS and Spitzer-IRAC data. The infrared extinction law is rederived throughout the disk and we found strong longitudinal variations in both diffuse and dense environments that we incorporate in our analysis.
\end{abstract}

Keywords. dust, extinction - Galaxy: disk - Galaxy: structure - infrared: ISM

Many Galactic dust studies to date have found an IR extinction law that is nearly constant and universal in the diffuse interstellar medium (ISM; e.g., Indebetouw et al. 2005), but more recent work has demonstrated that in regions of dense ISM, such as dark cores, the MIR $A_{\lambda}$ curve becomes shallower, likely due to dust grain growth (e.g., Weingartner \& Draine 2001). Thus, we questioned whether the Galactic extinction law would change substantially through the various ISM environments in the disk, beyond the simple, frequently-used "dense" / "diffuse" paradigm.

For this study, we combined photometry from the MIR Spitzer/IRAC surveys (Benjamin et al. 2003 ), spanning $\sim 150^{\circ}$ of nearly contiguous disk longitude, with the NIR 2MASS Catalogue (Skrutskie et al. 2006), to obtain a consistent set of photometric data in 7 bands $(1.2-8 \mu \mathrm{m})$. Using red clump stars, we have measured the relative extinction law along many lines of sight in the Galactic disk. We find strong, monotonic variations in $A_{\lambda} / A_{K s}$ as a function of galacto-centric angle, symmetric about the centre. This behaviour (a steepening extinction law at larger angles) persists even after the removal of known dense ISM, traced by ${ }^{13} \mathrm{CO}$ emission (GRS; Jackson et al. 2006), which suggests a secondary Galactic-scale dust property gradient (Zasowski et al. 2009).

We include these extinction law variations in a new technique for stellar reddening estimation, which uses long-baseline N/MIR colours to derive star-by-star extinction values more robustly than NIR-only techniques; in addition, this RJCE method (Rayleigh Jeans Color Excess method; Majewski, Nidever, \& Zasowski, in prep.) preserves stellar type information to create reliably-cleaned mid-plane colour-magnitude diagrams. The preservation of stellar type and luminosity class information permits 3-D mapping of the stars and the intervening dust without reliance on a static Galactic model.

\section{References}

Benjamin, R. A. et al. 2003, PASP, 115, 953

Indebetouw, R. et al. 2005, ApJ, 619, 931

Jackson, J. M. et al. 2006, ApJS, 163, 145

Skrutskie, M. F. et al. 2006, AJ, 131, 1163

Weingartner, J. C. \& Draine, B. T. 2001, ApJ, 548, 296

Zasowski, G. et al., 2009, ApJ, submitted 